\title{
Violência obstétrica: uma revisão integrativa
}

\author{
Obstetric violence: integrative review \\ Violencia obstétrica: una revisión integradora
}

\author{
Ana Clara Alves Tomé de Souza'; Pedro Henrique Campolina Silva Lucas"; Tahbatha Costa Lana ${ }^{\prime \prime \prime \prime}$; \\ Sheila Rubia Lindner'v; Torcata Amorim ${ }^{v}$; Mariana Santos Felisbino-Mendes ${ }^{V I}$
}

\begin{abstract}
RESUMO
Objetivo: revisar pesquisas brasileiras, identificando os tipos de violência obstétrica, possíveis causas observadas e o papel do enfermeiro nesse cenário. Método: revisão integrativa realizada em 2018, com artigos brasileiros selecionados na Biblioteca Virtual em Saúde. Resultados: revisados 16 artigos publicados entre 2004 e 2018. A violência obstétrica pode ser associada a: ofensa verbal e psicológica, expropriação do corpo feminino, privação de acompanhante, falta de informações, privação dos movimentos, banalização da dor e falta de privacidade. Possíveis causas: despreparo institucional e profissional, autoritarismo/hierarquização profissional, medicalização da assistência, nível socioeconômico e escolaridade das mulheres, e negação ou não reconhecimento da violência obstétrica. Conclusão: a enfermeira obstétrica pode contribuir para a redução dessa violência. São necessários mais investimentos na formação dessas profissionais e proporcionar assistência de qualidade no pré-natal e parto.
\end{abstract}

Descritores: Violência contra a mulher; parto humanizado; trabalho de parto; enfermagem obstétrica.

\section{ABSTRACT}

Objective: review Brazilian researches, identifying the types of obstetric violence, possible causes observed and the role of nurses in this scenario. Method: integrative review realized in 2018, with Brazilian articles selected from the Virtual Health Library. Results: obstetric violence can be associated with: verbal and psychological offense, expropriation of the female body, deprivation of companion, lack of information, deprivation of movement, trivialization of pain, and lack of privacy. Possible causes: institutional and professional unpreparedness, authoritarianism/professional hierarchy, medicalization of care, women's socioeconomic status and education, and denial or non-recognition of obstetric violence. Conclusion: the obstetric nurse can contribute to the reduction of this violence. More investments are needed in the formation of these professionals and provide quality assistance in prenatal and delivery obstetric.

Descriptors: Violence against women; humanizing delivery; labor, obstetric; obstetric nursing.

\section{RESUMEN}

Objetivo: revisar las investigaciones brasileñas, identificando los tipos de violencia obstétrica, las posibles causas observadas y el papel del enfermero en este escenario. Método: revisión integradora realizada en 2018, con artículos brasileños seleccionados de la Biblioteca Virtual en Salud. Resultados: la violencia obstétrica puede estar asociada con: ofensa verbal y psicológica, expropiación del cuerpo femenino, privación de compañero, falta de información, privación de movimiento, trivialización del dolor y falta de privacidad. Posibles causas: falta de preparación institucional y profesional, autoritarismo / jerarquía profesional, medicalización de la asistencia, nivel socioeconómico y escolaridad de las mujeres y negación o no reconocimiento de la violencia obstétrica. Conclusión: la enfermera obstétrica puede contribuir para la reducción de esta violencia. Se necesitan mas inversiones en la formación de estas profesionales y proporcionar una asistencia de calidad en prenatal y parto.

Descriptores: Violencia contra la mujer; parto humanizado; trabajo de parto; enfermería obstétrica.

\section{INTRODUÇÃO}

$O$ ato de partejar foi, até o século XIX, considerado uma atribuição feminina, realizada em sua maioria por parteiras ${ }^{1}$. Contudo, a partir da década de 1940 , do século XX, surge o processo de medicalização como consequência do significativo avanço tecnológico da medicina e assim, o parto passou a ser associado a um processo patológico, uma vez que acarretava riscos para a mulher ${ }^{2,3}$. Nesse contexto, "o corpo feminino era considerado imperfeito e por isso, incapaz de parir sem o auxílio das intervenções" 3:25.

'Enfermeira. Curso de Especialização em Enfermagem Obstétrica - Modalidade Residência, Universidade Federal de Minas Gerais. Brasil. E-mail: anaclaraa_19@hotmail.com

"Enfermeiro. Escola de Enfermagem, Universidade Federal de Minas Gerais. Brasil. E-mail: pedro_c lucas@yahoo.com.br

I'Enfermeira. Curso de Especialização em Enfermagem Obstétrica - Modalidade Residência, Universidade Federal de Minas Gerais. Brasil. E-mail: costa.tatha@gmail.com IVDoutora em Saúde Coletiva. Universidade Federal de Santa Catarina. Brasil. E-mail: sheila.lindner@gmail.com

voutora em Ciências. Curso de Especialização em Enfermagem Obstétrica - Modalidade Residência, Universidade Federal de Minas Gerais. Brasil. E-mail: torcata@enf.ufmg.br

v' Doutora em Enfermagem. Universidade Federal de Minas Gerais. Brasil. E-mail: marianafelisbino@yahoo.com.br 
Com isso, o parto deixou o ambiente domiciliar e passou a ocupar o espaço das instituições de saúde, conduzido por diferentes atores e submetendo as mulheres a diversos procedimentos em favor da ciência ${ }^{4}$. Os procedimentos invasivos e intervencionistas eram justificados por sua maior praticidade e por demandarem menor tempo ${ }^{5}$. Entretanto, resultaram na diminuição da autonomia da mulher e acarretaram maiores riscos para mãe e para o bebê ${ }^{1}$. Nesse contexto, as práticas intervencionistas começaram a ser realizadas rotineiramente ${ }^{6}$.

A Organização Mundial de Saúde (OMS) em 1996 dividiu essas práticas em quatro categorias: úteis e que devem ser estimuladas; práticas sem evidências para serem recomendadas; práticas prejudiciais ou ineficazes, que devem ser eliminadas da assistência ao parto (infusão intravenosa de rotina no trabalho de parto, o uso rotineiro de enema e tricotomia, dentre outras); e práticas utilizadas de modo inadequado como restrição hídrica e alimentar durante o trabalho de parto, uso liberal e rotineiro de episiotomia, parto operatório e exames vaginais repetidos ou frequentes ${ }^{6}$.

Dessa forma, a "imposição de intervenções danosas à integridade física e psicológica das mulheres no atendimento nas instituições, assim como, o desrespeito a autonomia da mulher" pode ser caracterizado como um ato de violência obstétrica ${ }^{7: 1}$.

No Brasil, apenas o estado de Santa Catarina possui uma lei que tipifica a violência obstétrica, considerando-a como "todo ato praticado pelo médico, pela equipe do hospital, por um familiar ou acompanhante que ofenda de forma verbal ou física, as mulheres gestantes, em trabalho de parto ou ainda, no período puerperal" ${ }^{8}$. Nos últimos anos, alguns estudos apontaram sobre os índices de práticas obstétricas desnecessárias no Brasil. Dados do Inquérito Nacional "Nascer no Brasil" mostram que apenas $5 \%$ dos partos vaginais ocorrem sem intervenções e revelam que o índice de cesarianas é de $52 \%$ do total de partos ${ }^{9}$, contrariando a recomendação da OMS de manter esses índices próximos de $15 \%{ }^{10}$. Além disso, $25 \%$ das mulheres relatam que sofreram algum tipo de agressão praticada por profissionais de saúde, durante a gestação ou no parto ${ }^{11}$.

Tendo em vista a violência obstétrica, associada à ausência de uma legislação específica e o índice elevado de práticas obstétricas desnecessárias, buscou-se com essa revisão conhecer os tipos de violência obstétrica e suas possíveis causas, observadas nas pesquisas brasileiras. Também buscou-se identificar como o papel do enfermeiro tem sido retratado neste cenário.

\section{METOdOLOGIA}

Trata-se de um estudo de revisão integrativa que buscou responder as questões norteadoras: Quais são os tipos de violência obstétrica e suas possíveis causas identificadas nas pesquisas brasileiras? Além de, como o papel do enfermeiro tem sido retratado nas pesquisas diante desse problema de saúde pública?

Para identificar os artigos de forma a responder as perguntas norteadoras dessa revisão, realizou-se o levantamento bibliográfico entre maio a setembro de 2018, no site da Biblioteca Virtual em Saúde (BVS). Utilizou-se como ponto de partida para a busca os descritores violência contra a mulher, "Parto Humanizado", "Trabalho de Parto", "Enfermagem Obstétrica", "Parto Obstétrico", “Obstetrícia”, "Enfermeira Obstétrica”, "Parto", "Gestante", "Gravidez", "Assistência ao parto", "Violência Institucional” e seus correspondentes em inglês e espanhol. Os critérios de inclusão para esta revisão foram estudos primários que abordassem o tema violência obstétrica no Brasil.

Foram encontradas 429 publicações, das quais foram excluídas 81 que não constituíam em artigos científicos. Dos 348, foram excluídos 126 artigos por se tratar da realidade de outros países, sobrando 222, dos quais 66 eram duplicados. Em seguida, realizou-se a leitura do resumo dos 156 artigos restantes, a partir da qual 37 artigos foram selecionados para leitura na íntegra e 120 foram excluídos, uma vez que 62 não abordavam nenhuma forma de violência e 58 abordavam outros tipos de violência. Após a leitura completa dos 37 artigos, 3 foram excluídos por não se tratar de estudos primários e 18 por abordar a violência obstétrica indiretamente.

A análise crítica final foi realizada com os 16 artigos selecionados, buscando identificar aspectos referentes à violência praticada por profissionais contra a mulher na assistência ao parto, com destaque para os tipos de violência, suas possíveis causas e como o papel do enfermeiro tem sido retratado nas pesquisas.

\section{RESULTADOS E DISCUSSÃo}

Os $16(100 \%)^{12-27}$ artigos selecionados foram publicados entre os anos de 2004-2018 e em revistas brasileiras, e são demonstrados na Figura 1. Observa-se que $6(37,5 \%)^{13,16,18,20-22}$ foram publicados em revistas de enfermagem, 2 $(12,5 \%)^{15,26}$ em revistas de saúde pública, e $8(50 \%)^{12,14,17,19,23-25,27}$ em outras revistas.

Após leitura e análise, observou-se que em todos os artigos as gestantes, parturientes, puérperas ou mulheres em situação de aborto foram vítimas de violência obstétrica praticada por profissionais de saúde e em um caso específico, pelos agentes penitenciários. 


\begin{tabular}{|c|c|}
\hline Títulos & $\begin{array}{l}\text { Ano de } \\
\text { publicação }\end{array}$ \\
\hline Para chegar ao Bojador, é preciso ir além da dor ${ }^{12}$ & 2015 \\
\hline Violência obstétrica na visão de enfermeiras obstetras ${ }^{13}$ & 2014 \\
\hline Violência institucional em maternidades públicas sob a ótica das usuárias ${ }^{14}$ & 2011 \\
\hline Violência institucional, autoridade médica e poder nas maternidades sob a ótica dos profissionais de saúde ${ }^{15}$ & 2013 \\
\hline Poder, violência e dominação simbólicas nos serviços públicos de saúde ${ }^{16}$ & 2004 \\
\hline O dilema de uma prática: experiências de aborto em uma maternidade pública de Salvador, Bahia ${ }^{17}$ & 2016 \\
\hline Do parto institucionalizado ao parto domiciliar ${ }^{18}$ & 2014 \\
\hline À margem da humanização? Experiências de parto de usuárias de uma maternidade pública de Porto Alegre-RS ${ }^{19}$ & 2017 \\
\hline Percepção de enfermeiras obstétricas acerca da violência obstétrica20 & 2018 \\
\hline Parto hospitalar: experiências de mulheres da periferia de Cuibá-MT21 & 2006 \\
\hline Percepção das mulheres sobre violência obstétrica ${ }^{22}$ & 2017 \\
\hline $\begin{array}{l}\text { Meio grogue e com as mãos amarradas: o primeiro contato com o recém-nascido segundo mulheres que } \\
\text { passaram por uma cesárea indesejada }{ }^{23}\end{array}$ & 2013 \\
\hline Nascer na prisão: gestação e parto atrás das grades no Brasil|24 & 2016 \\
\hline $\begin{array}{l}\text { Fatores associados à violência obstétrica na assistência ao parto vaginal em uma maternidade de alta } \\
\text { complexidade em Recife, Pernambuco } 25\end{array}$ & 2016 \\
\hline Desigualdades raciais, sociodemográficas e na assistência ao pré-natal e ao parto ${ }^{26}$ & 2005 \\
\hline Percepções de doulas naturólogas sobre gestação, parto e puerpério 27 & 2014 \\
\hline
\end{tabular}

FIGURA 1: Artigos incluídos no estudo e ano de publicação. Brasil, 2019.

Os tipos de violência no parto identificados nos artigos foram: ofensa verbal e psicológica $(11 \text { - 68,8\%) })^{12,14-24}$; expropriação do corpo feminino $\left(10\right.$ - 62,5\%) ${ }^{12,16,18-22,25-27}$; privação de acompanhantes $(7 \text { - 43,8\% })^{12,15,17,19,22-24}$; não fornecimento de informações $(8-50 \%)^{12,14,16,17,20-23}$, privação dos movimentos no trabalho de parto e parto $(6$ $37,5 \%)^{17,19,21-24}$; banalização da dor $(3-18,8 \%)^{14-16}$ e falta de privacidade $(2-12,5 \%)^{16,17}$.

Dentre as possíveis causas apontadas nos estudos destaca-se o despreparo profissional e institucional (12 $75 \%)^{12-18,20,21,23,25,26}$, medicalização da assistência $(10-62,5 \%)^{12-14,16,18-20,23,25,27}$, Autoritarismo/hierarquização profissional $(8-50 \%)^{14-16,18,19,21,23,25}$, nível socioeconômico e escolaridade $(5-43,8 \%)^{16,19,21,25,26}$ e por último, a negação ou não reconhecimento da violência obstétrica $(3-18,8 \%)^{14,15,20}$.

Apenas 4 (25\%) dos artigos ${ }^{13,18,20,25}$ discute sobre a importância do papel da enfermeira obstétrica e das obstetrizes no processo do parto, relatando que esta é uma profissional reconhecida mundialmente por estar capacitada e preparada para assistir a mulher neste momento.

Os achados dessa revisão integrativa apontam que a pesquisa sobre essa temática ainda é tímida no Brasil, sendo observado um pequeno avanço a partir do ano de 2013. Este tema ganhou visibilidade na segunda metade do século XXI, quando passou a ser mais estudado e a fazer parte de mostras artísticas, documentários, ações judiciais, dentre outros ${ }^{28}$. O termo violência obstétrica é utilizado para descrever os tipos de violência praticados por profissionais durante o cuidado obstétrico ${ }^{10,32}$. Contudo, o termo violência muitas vezes não é utilizado por receio de que os profissionais reajam de forma agressiva mediante a acusação de violência, sendo assim substituído por outros descritores, como por exemplo, humanização do parto ${ }^{28}$. Outros termos frequentemente utilizados como variação, são violência institucional e violência no parto. Essas variações podem estar atreladas ao fato da violência obstétrica ser um conceito recente, que foi difundido principalmente depois da mobilização em torno da humanização do parto e da publicação da Fundação Perseu Abramo "Mulheres brasileiras e gênero nos espaços públicos e privados", que evidenciou o tema violência no parto ${ }^{12}$.

Destaca-se que as mulheres, sendo elas gestantes, parturientes, em situação de abortamento ou puérperas, são vítimas frequentes da violência obstétrica. Entretanto, vê-se que o processo de parturição, é o momento em que mais se observa tal prática. Faz-se necessário destacar que a violência obstétrica é praticada por diferentes profissionais de saúde, gerando uma reflexão sobre a formação desses profissionais ${ }^{13,36}$.

\section{Tipos de violência obstétrica}

O mau atendimento nos serviços de saúde é retratado por muitas mulheres como um ato de violência ${ }^{14}$. Em alguns estudos as parturientes relataram que não foram informadas sobre as intervenções realizadas e com isso, se sentiram objetificadas, por não terem o direito de se expressarem ${ }^{15}$. Além de não explicarem os procedimentos, muitos profissionais nem sequer se apresentaram à paciente ${ }^{16}$. Algumas usuárias descreveram os partos como violentos e que 
muitas vezes os funcionários tinham posturas agressivas e intimidadoras ${ }^{17}$. Nos estudos analisados, foram identificados também que a falta de informação faz com que a mulher imagine que todos os procedimentos realizados se configurem como de rotina e isso, leva a expropriação do corpo feminino ${ }^{18}$. A mulher deixa de acreditar em seu próprio corpo e começa a acreditar que as intervenções são realizadas para salvar a sua vida e a de seu bebê ${ }^{14,19,20}$.

Percebe-se que os tipos de violência obstétrica relatados não são caracterizados somente por procedimentos técnicos, mas também pelo o uso de frases ofensivas, repreensões, ameaças contra as mulheres e seus bebês no momento do parto. Alterações do volume de voz e o uso de palavras que causam humilhação, são apontadas como frequentes e até mesmo consentidas pelos profissionais, sendo classificados como violência verbal e psicológica ${ }^{20,22,33}$. Destaca-se ainda a utilização de jargões pejorativos e piadas, como exemplo: "Na hora de fazer não chorou, está chorando agora por quê?", "ah, não chora não, ano que vem você está aqui de novo"15:2292, ou como no caso de uma paciente em situação de aborto que, ao ser examinada ouviu a seguinte pergunta: "Quem quer um feto morto?"17:52. Apesar de vários profissionais desaprovarem essa ação, observa-se a banalização do uso desses jargões, que comumente estão presentes nesta assistência ${ }^{15}$.

Para as mulheres, o processo do parto está diretamente associado a dor e ao sofrimento, sendo estes, outros fatores associados à violência durante a assistência obstétrica ${ }^{12,21,30}$. Dessa forma, se calar diante da dor é um recurso constantemente adotado pelas parturientes para tentar não sofrer algum tipo de violência, uma vez que foi observado que, quando a mulher expressa seu sofrimento, ela passa a ser tratada com descaso pelos profissionais ${ }^{14}$, incluindo frequentes ameaças de abandono ${ }^{15}$. Muitas mulheres se tornam vítimas da violência obstétrica por temerem que a reclamação possa ser mal interpretada pelo profissional, refletindo de forma negativa na assistência prestada tanto a elas quanto ao bebêt ${ }^{14}$.

Além do suporte emocional, o acompanhante pode auxiliar na evolução do trabalho de parto e aumentar a sensação de controle da paciente, resultando em menores taxas de intervenções nesse momento, diminuindo o uso de analgesia e aumentando a probabilidade do parto vaginal espontâneo ${ }^{22,30}$. Entretanto, em várias instituições, a presença do acompanhante ainda não é permitida. Em um dos estudos desta revisão, o índice de acompanhantes alcançou somente $3 \%{ }^{22}$. Dessa forma, a restrição de um acompanhante representa não só um ato de violência obstétrica, mas o descumprimento da lei $n^{\circ} 11.108$, conhecida como lei do acompanhante ${ }^{22,23}$.

Somados à violência verbal e psicológica se encontra a violência física, sendo identificada nos estudos como amarrar as parturientes durante as cesarianas ${ }^{17,23}$ e contenção durante o parto, com a justificativa de que a mulher não quis ficar na posição que o profissional solicitou ${ }^{21}$. Inquérito nacional revelou que $36 \%$ das gestantes privadas de liberdade foram algemadas durante a internação e $8 \%$ durante o trabalho de parto e parto ${ }^{24}$. Buscando combater este ato, foi promulgada em abril de 2017 a Lei no 13.434 que proibiu o uso de contenção por algemas em gestantes e parturientes durante a assistência obstétrica ${ }^{8}$.

Acrescenta-se ainda, a tricotomia, privação de alimento, administração deliberada de ocitocina, amniotomia precoce de rotina, adoção da posição supina, o incentivo aos puxos voluntários (manobra de Valsalva), o Kristeller e a episiotomia de rotina identificadas como práticas prejudiciais e que devem ser eliminadas ou que não devem ser estimuladas, por não existir evidências suficientes ${ }^{6}$. A realização desses procedimentos evidenciam a violência sofrida pelas mulheres durante o processo de parturição. O parto é um evento fisiológico, que ao sofrer intervenção, além de ocasionar repercussões físicas para a parturiente, acabam apagando expectativas e sonhos. ${ }^{33}$

\section{Possíveis causas para violência obstétrica}

Observou-se que o despreparo institucional e a formação profissional foram os temas mais abordados nos estudos analisados. As jornadas intensas de trabalho, associadas aos recursos humanos e materiais precários, também são consideradas possíveis causas para violência praticada contra as pacientes durante o trabalho de parto e parto ${ }^{13,15}$.

O conhecimento limitado sobre a prática baseada em evidências faz com que mulheres sejam objetificadas para treinamentos de internos em procedimentos como episiotomia, fórceps e até mesmo cesarianas, favorecendo a prática da violência obstétrica ${ }^{28}$. Esse cenário tem sido sustentado por um sistema de formação e de saúde deficientes e pela falta de fiscalização adequada dessas instituições ${ }^{13}$.

Outro ponto levantado nos estudos diz respeito ao autoritarismo ou hierarquização profissional. Esse tipo de conduta também contribui para a expropriação do corpo feminino e o uso da medicalização do parto, uma vez que os profissionais usam/abusam de sua formação para interferir com condutas muitas vezes desrespeitosas, não atendendo os desejos da mulher ${ }^{19,25,35}$. As parturientes ficam submissas a condutas que elas não entendem, mas acreditam que é para o bem e aceitam, mesmo que sobre pressão ${ }^{19}$. Muitos profissionais não reconhecem a violência obstétrica, e quando há uma prática que é considerada prejudicial para a paciente, se justificam como necessária para o binômio 20,34 . 
O nível socioeconômico também é um fator identificado nos estudos como predisponente à violência obstétrica, uma vez que mulheres negras, com menor renda e nível de escolaridade, são alvo mais fácil para a prática da violência, como a negação de acompanhantes e o uso de condutas rotineiras ${ }^{19,26}$. Foi identificado ainda que mulheres com níveis socioeconômicos mais baixos e de baixa escolaridade tiveram menor acesso ao pré-natal, o que contribui para a falta de informação sobre o trabalho de parto e parto ${ }^{26}$.

Esses apontamentos dos estudos evidenciam a mudança na formação dos profissionais de saúde como forma de superação do quadro de violência obstétrica, sendo identificada a necessidade de modificações na grade curricular dos cursos de graduação, para que a assistência seja pautada em evidências científicas e com reflexão crítica sobre as intervenções realizadas ${ }^{13,33}$. Evidencia-se também a importância da participação em congressos, cursos de reciclagem e oficinas para constante atualização ${ }^{18}$.

Diante desse cenário destaca-se o preparo para o parto, que deve ser realizado desde a primeira consulta de pré-natal. Esta ação contribui para que as mulheres, ao chegarem à maternidade, estejam melhor informadas e de certo modo, mais preparadas tanto fisicamente quanto psicologicamente para o trabalho de parto e parto, reconhecendo seus direitos e deveres e sabendo sobre o processo de parturição ${ }^{13,32}$.

\section{Importância da enfermagem obstétrica nesse cenário}

Poucos artigos abordaram a importância da enfermagem obstétrica no contexto da violência obstétrica. Os cinco que o fizeram destacaram a importância desse profissional para redução de eventos traumáticos na vida das mulheres $^{13,18,20}$, para a construção de um modelo humanizado ${ }^{20,26}$ e para utilização das boas práticas ${ }^{16,18}$.

Sabe-se que a enfermagem trabalha com o cuidado e respeita os processos fisiológicos e naturais da parturiente, contribuindo assim para redução de práticas intervencionistas desnecessárias e consequente desmedicalização do parto.13,29,31 Acredita-se ainda que a Enfermagem Obstétrica possa proporcionar grandes mudanças em relação às práticas violentas cometidas na assistência ao parto ${ }^{13}$, uma vez que o papel desta profissional já é reconhecido mundialmente, em especial, por ser capacitada e com melhor custo-benefício para prestar a assistência a parturiente e ao bebê.

Quando comparado a outros modelos, o cuidado ofertado pelas enfermeiras obstetras apresenta diversos benefícios como a redução do uso de anestesia peridural, de episiotomia e de parto instrumental (com fórceps ou vácuo extrator), bem como, maior chance de parto vaginal espontâneo, de iniciar o aleitamento materno precocemente e de satisfação materna ${ }^{18}$. Esta profissional pode contribuir também para reduzir o número de cesáreas sem indicação real e contribuir positivamente com os indicadores de morbimortalidade materna e perinatal ${ }^{31}$.

Dessa forma, faz-se necessário fortalecer a formação da enfermagem obstétrica no país, para evitar a mera reprodução de um modelo tecnicista e profissional centrado que ainda resiste, além de garantir alguns dos princípios preconizados pelo SUS que são a equidade e a integralidade ${ }^{13,20}$.

Ademais, a construção de Centros de Parto Normais (CPN), o parto domiciliar e uma maior atuação de enfermeiras obstétricas representam alternativas para a mudança do quadro de violência na assistência ao parto excessivamente intervencionista ${ }^{13,15}$. Nos Centros de Parto Normal a parturiente tem a liberdade de ter um acompanhante de sua escolha e uma doula, além de poder escolher a posição que desejar até o nascimento do bebê. Estes espaços apresentam resultados perinatais significativamente positivos em relação aos partos normais e de risco habitual ${ }^{25,27}$.

Soma-se a isso que, esse tipo de assistência promove a compreensão da fisiologia da mulher e que, o estado psicológico e emocional, interferem diretamente durante o trabalho de parto. Acrescenta-se também o respeito às atitudes de cada parturiente e o uso das práticas baseadas em evidências pois, possuem relação direta com a segurança e com os resultados positivos para o binômio ${ }^{13,18}$. Esse tipo de assistência tem mostrado maior satisfação da mulher e de seus familiares ${ }^{18}$.

Adicionalmente, vê-se que grande parte dos estudos foi publicada em revistas de enfermagem. Isto mostra que apesar dos poucos resultados obtidos, a enfermagem tem contribuído para maior evidência do tema e da construção do conceito da violência obstétrica no país.

\section{CONCLUSÃO}

Nessa revisão integrativa foram revisados 16 artigos publicados em periódicos brasileiros no período de 2004 a 2018 e observou-se que os estudos utilizam diferentes terminologias como: violência obstétrica, violência institucional ou violência no parto, para abordar a violência sofrida por mulheres no ciclo gravídico puerperal. A ofensa verbal e psicológica, expropriação do corpo feminino, privação de acompanhante, falta de informações, privação dos movimentos, banalização da dor, e falta de privacidade são alguns tipos de violência relatados nos estudos, elucidando a violência no parto como prática frequente e pouco retratada no cenário brasileiro. 
Ressalta-se que o despreparo profissional, a medicalização, o autoritarismo/hierarquização profissional e nível socioeconômico das mulheres estão interligados e atuam sinergicamente, sendo destacados como causas importantes para a violência obstétrica.

Mudanças nesse cenário estão atreladas a políticas públicas, com destaque para formação profissional, em especial das enfermeiras obstétricas, cujo papel primordial é o fortalecimento do modelo humanístico, buscando o respeito, a fisiologia do parto e o protagonismo da mulher. Destaca-se também a importância da realização de um pré-natal de qualidade, visando informar e preparar física e psicologicamente a mulher para o trabalho de parto e parto.

\section{REFERÊNCIAS}

1. Wolff LR, Waldow VR. Consented violence: women in labor and delivery. Saude soc. (online). 2008 [cited 2019 Jul 15]; 17(3):138-51. DOI: https://doi.org/10.1590/S0104-12902008000300014.

2. Moura FMJSP, Crizostomo CD, Nery NI, Mendonça MRC, Araújo OD, Rocha SS. Humanization and nursing assistance to normal childbirth. Rev. bras. enferm. 2007 [cited 2019 Jul 15]; 60(4):452-55. DOI: https://doi.org/10.1590/S0034-71672007000400018.

3. Vilanova AKL, Carvalho FS, Portela NLC, Paes ARM, Santos DAS. Percepções da parturiente acerca da presença do acompanhante no processo de parturição. Rev. Augustus. 2015 [cited 2019 Jul 15]; 20(39):24-37. DOI: http://dx.doi.org/10.15202/10.15202/19811896.2015v20n39p24.

4. Santos RAA, Melo MCP, Cruz DD. Trajetória de humanização do parto no Brasil a partir de uma revisão integrativa de literatura. Cad. Cult. Ciênc. 2015 [cited 2019 Jul 07]; 13(2). Available from: https://www.researchgate.net/publication/276880008_TRAJETORIA_DE_HUMANIZACAO_DO_PARTO_NO_BRASIL_A_PARTIR_ DE_UMA_REVISAO_INTEGRATIVA_DE_LITERATURA

5. Viana LVM, Ferreira KM, Mesquita MASB. Humanization normal child birth: a review of literature. Rev. Saúde em Foco. 2014 [cited 2019 Oct 07]; 1(2):134-48. Available from: http://www4.fsanet.com.br/revista/index.php/saudeemfoco/article/view/245/431

6. Silva JK, Mercês MC, Messias JMA, Guimarães KP, Jesus LMSB. Obstetric violence within the hospital environment: experience report on inconsistencies and controversies. Rev. enferm. UFPE on line. 2015 [cited 2019 Oct 20]; 9(12):1345-51. Available from: https://periodicos.ufpe.br/revistas/revistaenfermagem/article/view/10843

7. Muniz BMV, Barbosa RM. Questioning the care in childbirth: is it care or violence? In: Memorias convención internacional de salud pública. Cuba Salud, 2012. [cited 2019 Oct 20]. Available from:

http://www.convencionsalud2012.sld.cu/index.php/convencionsalud/2012/paper/view/744/321

8. Lei no 17.097. Dispõe sobre a implantação de medidas de informação e proteção à gestante e parturiente contra a violência obstétrica no Estado de Santa Catarina, 17 de janeiro de 2017. [cited 2019 Oct 22]. Available from: http://leis.alesc.sc.gov.br/html/2017/17097_2017_lei.html

9. Leal MC, Pereira APE, Domingues RMSM, Theme Filha MM, Dias MAB, Nakamur PM. Obstetric interventions during labor and childbirth in Brazilian low-risk women. Cad. Saúde Pública (Online). 2014 [cited 2019 Oct 22]; 30(17):47. DOI: https://doi.org/10.1590/0102-311X00151513.

10. Parto do princípio - Mulheres em rede pela maternidade ativa. Dossiê da Violência Obstétrica: "Parirás com dor", 2012 [cited 2019 Oct 22]. Available from: https://www.senado.gov.br/comissoes/documentos/SSCEPI/DOC\%20VCM\%20367.pdf

11. Mulheres brasileiras e gênero nos espaços público e privado: pesquisa de opinião pública, Fundação Perseu Abramo, 2010 [cited 2019 Nov 10]. Available from: http://csbh.fpabramo.org.br/sites/default/files/pesquisaintegra.pdf

12. Carneiro R. "To go past the Bojador, one must go beyond pain": labor suffering and its potencial. Revista Latinoamericana. 2015 [cited 2019 Nov 10]; 20: 91-112. DOI: https://doi.org/10.1590/1984-6487.sess.2015.20.08.a.

13. Silva MG, Marcelino MC, Rodrigues LSP, Toro RC, Shimo AKK. Obstetric violence according to obstetric nurses. Revista Rene. 2014 [cited 2019 Nov 10]; 15(4):720-8. DOI: https://doi.org/10.15253/2175-6783.2014000400020.

14. Aguiar JM, D'oliveira AFPL. Institutional violence in public maternity hospitals: the women's view. Interface - Comunic., Saude, Educ. 2011 [cited 2019 Nov 10]; 15(36):79-91. DOI: https://doi.org/10.1590/S1414-32832010005000035.

15. Aguiar JM, D'oliveira AFPL, Schraiber LB. Institutional violence, medical authority, and power relations in maternity hospitals from the perspective of health workers. Cad. Saúde Pública (Online). 2013 [cited 2019 Nov 10]; 29(11):2287-96. DOI: https://doi.org/10.1590/0102-311x00074912.

16. Pereira WR. Power, violence and symbolic domination in public health services. Texto \& contexto enferm. 2004 [cited 2019 Dec 11]; 13(3):391-400. DOI: https://doi.org/10.1590/\$0104-07072004000300008.

17. Mccallum C, Menezes G, Reis AP. The dilemma of a practice: experiences of abortion in a public maternity hospital in the city of Salvador, Bahia. Hist. cienc. saude-Manguinhos. 2016 [cited 2019 Nov 11]; 23(1):37-56. DOI: https://doi.org/10.1590/S010459702016000100004.

18. Sanfelice CFO, Abbud FSF, Pregnolatto OS, Silva MO, Shimo AKK. From institutionalized birth to home birth. Revista Rene. 2014[cited 2019 Nov 11]; 15(2):362-70. DOI: https://doi.org/10.15253/2175-6783.2014000200022.

19. Pedroso CNLS, López LC. Apart from humanization? Birth experiences of users of a public maternity hospital in Porto Alegre-RS, Brazil. Physis (online). 2017 [cited 2019 Nov 15]; 27(4):1163-84. DOI: https://doi.org/10.1590/s0103-73312017000400016.

20. Leal SYP, Lima VLA, Silva AF, Soares PDFL, Santana LR, Pereira A. Perception of nurse midwives on obstetric violence. Cogitare enferm. Rev. 2018 [cited 2019 Nov 15]; 23(2). DOI: http://dx.doi.org/10.5380/ce.v23i1.52473. 
21. Teixeira NZF, Pereira WR. Hospital delivery - women's experience from the suburbs of Cuibá-MT. Rev. bras. enferm. (Online). 2006 [cited 2019 Nov 15]; 59(6):740-44. DOI: https://doi.org/10.1590/S0034-71672006000600004.

22. Oliveira TR, Costa REOL, Monte N, Veras JMMF, Sá MIMR. Women's perception on obstetric violence. Rev. enferm. UFPE on line. 2017 [cited 2019 Nov 15]; 11(1):40-46. Available from: https://periodicos.ufpe.br/revistas/revistaenfermagem/article/view/11876.

23. Salgado HO, Niy DY, Diniz CSG. Groggy and with tied hands: the first contact with thenewborn according to women that had an unwantedc-section. Rev. bras. crescimento desenvolv. hum.(online). 2013 [cited 2019 Nov 15]; 23(2):190-97. Available from: http://pepsic.bvsalud.org/pdf/rbcdh/v23n2/pt_11.pdf.

24. Leal MC, Ayres BVS, Esteves PAP, Sánchez AR, Larouzé B. Birth in prison: pregnancy and birth behind bars in Brazil. Ciênc. saúde coletiva. 2016 [cited 2019 Nov 16]; 21(7):2061-70. DOI: https://doi.org/10.1590/1413-81232015217.02592016.

25. Andrade PON, Silva JQP, Diniz CMM, Caminha MFC. Factors associated with obstetric abuse invaginal birth care at a highcomplexitymaternity unit in Recife, Pernambuco. Rev. Bras. Saúde Matern. Infant.(online). 2016 [cited 2019 Nov 16]; 16(1):2937. DOI: http://dx.doi.org/10.1590/1806-93042016000100004.

26. Leal MC, Gama SGN, Cunha CB. Racial, sociodemographic, and prenatal andchildbirth care inequalities in Brazil, 1999-2001. Rev. saúde pública (Online). 2005 [cited 2019 Nov 16]; 39(1):100-07. DOI: https://doi.org/10.1590/S0034-89102005000100013.

27. Souza SV, Scheid AO. Doulas naturólogas perceptions about pregnancy, birth and puerperium. Cad. naturol. terap. complem. 2014 [cited 2019 Nov 16]; 3(4). DOI: http://dx.doi.org/10.19177/cntc.v3e4201443-52.

28. Diniz SG, Salgado HO, Andrezzo HFA, Carvalho PGC, Carvalho PCA, Aguiar CA, et al. Abuse and disrespect in childbirth care as a public health issue in Brazil: origins, definitions, impacts on maternal health, and proposals for its prevention. Jornal of Human Growth and Development. 2015 [cited 2019 Nov 17]; 25(3):377-84. DOI: http://dx.doi.org/10.7322/jhgd.106080.

29. Vargens $O M C$, Nunes $S$, Silva CM, Progianti JM. Invasive procedures in midwifery care from a gender perspective. Rev enferm UERJ. 2016 [cited 2019 Dec 13]; 24(6):e15066. DOI: http://dx.doi.org/10.12957/reuerj.2016.15066.

30. Oliveira LLF, Trezza MCSF, Santos AAP, Melo GC, Sanches METL, Pinto LMTR. The experiences of comfort and discomfort of woman in labor and childbirth. Rev enferm UERJ. 2017 [cited 2019 Dec 13]; 25:e14203. DOI: https://doi.org/10.12957/reuerj.2017.14203.

31. Sanches METL, Barros SMO, Santos AAP, Lucena TS. Obstetric nurse's role in the care of labor and chilbirth. Rev enferm UERJ. 2019 [cited 2019 Dec 13]; 27:e43933. DOI: https://doi.org/10.12957/reuerj.2019.43933.

32. Carniel F, Vital DS, Souza TDP. Routine episiotomy: need versus obstetric violence. J. nurs. health. 2019 [cited 2019 Dec 13]; 9(2):e199204. DOI: http://dx.doi.org/10.15210/jonah.v9i2.14425.

33. Kopereck CS, Matos GC, Soares MC, Escobal APL, Quadro PP, Cecagno S. Obstetric violence in the multinational context. Rev. enferm. UFPE on line. 2018 [cited 2019 Dec 13]; 12(7):2050-60. DOI: https://doi.org/10.5205/1981-8963-v12i7a231399p20502060-2018.

34. Inagaki ADM, Lopes RJPL, Cardoso NP, Feitosa LM, Abud ACF, Ribeiro CJN. Factors associated with the humanization of care in a public maternity. Rev. enferm. UFPE on line. 2018 [cited 2019 Dec 13]; 12(7):1879-86. DOI: https://doi.org/10.5205/1981-8963v12i7a231395p1879-1886-2018.

35. Rodrigues DP, Alves VH, Vieira RS, Leão DCMR, Paula E, Pimentel MM. Obstetric violence in the context of labor and childbirth. Rev. enferm. UFPE on line. 2018 [cited 2019 Dec 13]; 12(1):236-46. DOI: https://doi.org/10.5205/1981-8963-v12i1a23523p236246-2018.

36. Oliveira MSS, Rocha VSC, Arrais TMSN, Alves SM, Marques AA, Oliveira DR, et al. Experiences of obstetric violence experienced by parturientes. ABCS Health Sci. 2019 [cited 2019 Dec 13]; 44(2):114-19. DOI: https://dx.doi.org/10.7322/abcshs.v44i2.1188. 